\title{
Acurácia das semeadoras-adubadoras à taxa variável
}

\author{
Accuracy of variable rate planters \\ Otávio Dias da Costa Machado ${ }^{\mathrm{I}}$ Airton dos Santos Alonço ${ }^{\mathrm{II}}$ \\ Mateus Potrich BelléII Cristian Josue Franck ${ }^{I I}$ \\ - REVISÃO BIBLIOGRÁFICA -
}

\section{RESUMO}

As semeadoras-adubadoras à taxa variável (TV) estão presentes em estudos focados em atributos do sistema de produção, fazendo com que o conhecimento desenvolvido acerca da qualidade da dosagem dessas máquinas esteja ainda incipiente. No entanto, os dados disponíveis podem ser utilizados para aprofundar a discussão da acurácia, que é o erro percentual entre a dose planejada e a obtida. Dessa maneira, procedeu-se a uma avaliação a partir da meta-análise de dados das publicações oficiais, qualificando a acurácia da dosagem de sementes em diferentes culturas. Dentre os experimentos disponiveis, apenas em um caso a acurácia manteve-se abaixo do valor máximo recomendado de 3\%; com operação estável e sem tendências de distorção da dosagem ao longo das densidades testadas, apresentando ainda 99\% de variação explicada e inclinação 0,95. Nos demais referenciais, a qualidade operacional foi degradada por subdosagens crescentes ao longo das doses, com inclinações das lineares que reduziram-se até 0,58 e com acurácias de até $34,75 \%$. Apesar disso, as distribuições mantiveram coeficientes de determinação entre 97 a 99\%, indicando possiveis efeitos sistemáticos e proporcionais do algoritmo ou calibração dos equipamentos. Independente da cultura, as semeadoras aumentaram a acurácia com o aumento da dose, apresentando regiões de melhor desempenho operando em menores niveis.

Palavras-chave: agricultura de precisão, semeadoras em linha de precisão, controladores.

\section{ABSTRACT}

Variable-rate planters are present in experiments that had focused in crop production attributes; maintain the quality of dosage still unacquainted. However, available data could be used to discuss machine accuracy, which is the percentage error between the planned and obtained dosages. Thus, was proceeded an assessment of accuracy from the meta-analysis of data from official publications, describing the operation in different cultures. Among the available experiments, only in one case the accuracy remained below the maximum recommended value of 3\%; with stable operation without distorting tendencies of dosage over the tested densities, with the distribution of the measurements with $99 \%$ explained variation and slope of 0.95. In other benchmarks, operational quality has been degraded by under dosing when increasing doses, with linear slopes which were reduced to 0.58 and accuracies up to $34.75 \%$. Nevertheless, the distribution maintains coefficients between 97 to $99 \%$, which indicates that there may have been happened systematic and proportional effects of the equipment algorithm or calibration. Regardless of the crop, planters had increased accuracy along increasing dosages, showing the regions with the best performance on lower populations.

Key words: precision agriculture, seeders, control systems.

\section{INTRODUÇÃO}

A Agricultura de Precisão (AP), segundo MANTOVANI (2000), é um conceito de condução de sistemas agrícolas consolidado em tecnologias atuais para o manejo do solo, insumos e culturas, capaz de permitir a identificação de variações espaciais e temporais de atributos que afetam a produtividade. STAFFORD (2000) relatou que sua aplicação tem gerado vantagens competitivas para os agricultores, pois a evolução do geo-referenciamento, dos sistemas de informação geográfica e do controle de máquinas agrícolas permitiu entender e intervir nos

\footnotetext{
Instituto Federal de Educação Ciência e Tecnologia do Rio Grande do Sul (IFRS), Campus Bento Gonçalves, Avenida Osvaldo Aranha, 540, 95700-000, Bento Gonçalves, RS, Brasil. E-mail: otavio.machado@bento.ifrs.edu.br. Autor para correspondência.

IILaboratório de Pesquisa e Desenvolvimento de Máquinas Agrícolas (Laserg), Universidade Federal de Santa Maria (UFSM), Santa
} Maria, RS, Brasil. 
cultivos em escala localizada. Segundo o autor, o desenvolvimento de máquinas agrícolas capazes de executar intervenções à Taxa Variável (TV) foi parte essencial no sistema, pois a aplicação correta potencializa os efeitos de todos os levantamentos de atributos e dos recursos tecnológicos utilizados até obterem-se as prescrições. Em contrapartida, FULTON et al. (2003) afirmaram que as limitações e erros da tecnologia de aplicação à TV são geralmente desconhecidos.

No início da utilização de operações agrícolas com variação espacial, foi relatado por SCHUELLER (1996) que a técnica estava se caracterizando como uma tendência, com pesquisadores e produtores entusiasmados com seus conceitos. Havia uma atratividade para os aspectos tecnológicos, mas sobretudo porque a TV inseria as propriedades no modelo dos sistemas de produção contemporâneos e modernos. Entretanto, o autor identificou limitações para a expansão da técnica, dentre elas, a acurácia das máquinas, que é o erro percentual da dosagem de um insumo; o que, de acordo com o sistema de controle, relaciona uma dosagem experimental com uma meta prescrita por um mapa, comando a partir de um sensor ou valor configurado pelo operador na interface do controlador.

A TV de sementes e fertilizantes em linha é recente no mercado sul-americano, mas os dados disponíveis da Argentina demonstram que o número de equipamentos tem aumentado de 40 a $60 \%$ ao ano desde 2005 (MÉNDEZ, 2011). Nas condições norteamericanas, o mercado destas semeadoras também tem aumentado (FULTON, 2009a), estando presentes em $30 \%$ das propriedades da região do cinturão do milho (PASZKIEWICZ \& BUTZEN, 2007). Nos Estados Unidos, a TV irá tornar-se standard para as semeadoras, bem como para os distribuidores de insumos, de acordo com as estimativas de HAAG \& STAGGENBORG (2006).

No mercado brasileiro, uma das primeiras referências do uso de semeadoras-adubadoras à TV, é observada no experimento de MOLIN et al. (2006). Essas pesquisas e suas sucessoras vêm tendo como meta o estudo de efeitos no sistema de produção, não havendo uma discussão detalhada do desempenho das máquinas.

Visando aprofundar a discussão da acurácia, os dados de aferições de populações de cultivos agrícolas implantados com semeadorasadubadoras à TV disponíveis em publicações oficiais, foram submetidos a uma meta-análise, discutindo comparativamente o desempenho e as condições em que ocorrem distorções na dosagem. A análise da correlação entre as dosagens prescritas e experimentais foi adotada, embasada na metodologia e valores referenciais de SCHUMANN et al. (2006).

Agricultura de Precisão e Taxa Variável

A AP é um sistema gerencial complexo, capaz de identificar atributos espacialmente, mas também quanto às suas alterações ao longo dos ciclos agrícolas. Por isso, considera-se que a técnica é capaz de mapear as glebas agrícolas quanto as suas variabilidades espacial e temporal. Segundo AUERNHAMMER (2001), a AP foi impulsionada pela aplicação de fertilizantes, limitando seu potencial. $\mathrm{O}$ autor estimou que a técnica seria mais valorizada quando passasse a ser reconhecida pelos seus benefícios adicionais, do aumento do registro, fluxo e controle da informação e ainda pela redução do impacto ambiental.

Ao desenvolver meios de identificar a variabilidade, aplica-se um conjunto de processos para definição da prescrição e realização das intervenções. As ações precedem, gerenciam, executam e monitoram as operações agrícolas. Essas técnicas recebem diferentes denominações, sendo bastante conhecida na literatura internacional a sigla VRT - Variable-Rate Technology (ANDRADESANCHEZ \& HEUN, 2010) ou VRA - Variable Rate Application (GRISSO et al., 2011). Os termos 'aplicação em dose variável' ou 'aplicação à taxa variável', também podem ser encontrados, mas foram mais adequados aos primórdios da técnica, quando concentrava-se na distribuição de insumos. Entretanto, tecnicamente, o termo aplicação não é adequado para referenciar algumas operações agrícolas, dentre elas a semeadura, sendo o conceito Taxa Variável - TV - associado com a respectiva operação, mais coerente (BERNARDI et al., 2004).

$O$ surgimento da TV ocorreu na agricultura norte-americana, na década de 1970 . Então utilizando aplicações contínuas, visando a eliminar variações durante pulverizações, sistemas de controle e válvulas foram desenvolvidos para compensar efeitos das trocas de velocidade de trabalho na vazão, permitindo pulverizações homogêneas (GEBHARDT et al., 1974; VIDRINE et al., 1975). O desenvolvimento dessas máquinas permaneceu latente, até que os microcomputadores, sensores e sistemas de posicionamento estivessem disponíveis e em custos acessíveis. Dessa forma, as máquinas evoluíram sensivelmente com a adoção dos Sistemas Globais de Navegação por Satélite (GNSS) e da porta de comunicação serial RS-232, que passou a permitir a transferência de mapas de 
prescrição dos computadores para os controladores (SHEARER et al., 2002). A técnica está atualmente bastante disseminada nas condições norteamericanas, pois, segundo FULTON et al. (2010), a AP e a TV são práticas comuns em muitas das propriedades agrícolas.

Quanto ao potencial da TV, os benefícios de adequação da aplicação de insumos no manejo das culturas incluem aperfeiçoar o uso dos recursos, melhorar a eficácia do equipamento e do insumo e aumentar a rentabilidade. Do ponto de vista ambiental, permite minimizar sobreaplicações e evitar aplicações em áreas protegidas. O controle de unidades ativas ou seções pode melhorar a eficiência e acurácia da aplicação, reduzir a fitotoxicidade, otimizar a jornada do operador e reduzir a fadiga (FULTON, 2009b).

A execução das operações à TV predomina com a utilização de prescrições (mapbased), as quais representam a espacialização das dosagens previamente planejadas e transferidas para o controlador. Nessas operações, a arquitetura do sistema de controle é em malha fechada, com um ciclo de sinais em que o acionamento dos atuadores é monitorado, retornando ao início do processo como um valor de correção. Para elaborar as prescrições, são analisados dados de produtividades e indicadores das culturas em ciclos anteriores, propriedades físicas e químicas do solo, previsões climáticas, de rendimento e de mercado. Atualmente, podem ser utilizados dados obtidos por sensores, tais como da eletrocondutividade do solo. Complexos sistemas computacionais são empregados para $o$ processamento desses dados e estudo de relações, para as tomadas de decisão que indicam qual dose será a mais adequada em cada ponto. São utilizados aplicativos computacionais de banco de dados, planilhas, sistemas de informação geográfica e outros (GRISSO et al., 2009; PING et al., 2008).

Está sendo também utilizada a TV em tempo real (sensor-based), que não necessita elaboração de mapas, reduzindo expressivamente a complexidade da preparação gerencial. A partir de leituras de atributos por sensores, uma rotina de cálculos do algoritmo do sistema de controle define uma prescrição e executa um comando para os atuadores que controlam a dosagem, durante a operação no campo. Não é necessária, obrigatoriamente, a utilização de sistemas de localização e a malha dos sistemas de controle é fechada (GRISSO et al., 2009).

Em um terceiro tipo de sistema, a dose é selecionada pelo operador, no terminal que serve de interface. Por não haver um ciclo de sinais, a arquitetura é denominada malha aberta, ou seja, não há mensuração do acionamento do atuador e o comando da dosagem provindo do controlador não sofre correção. A acurácia é dependente do valor de comando que o algoritmo atribui a cada dose, durante o processo de calibração. Limitações operacionais são decorrentes da necessidade de habilidade suficiente para identificar o fator de mudança e alterar a dosagem proporcionalmente, bem como manter a qualidade do procedimento ao longo de um turno de trabalho (GRISSO et al., 2009; FULTON, 2009a). A operação por comando do operador com semeadora-adubadora à TV tem exemplo em MOLIN et al. (2006), em que a máquina operou com quatro densidades na semeadura de um experimento de milho.

Os controladores das semeadoras à TV têm oferecido as duas opções de arquitetura no mesmo equipamento, sendo possível utilizá-las tanto com mapas quanto pela seleção da dosagem na interface. A TV em tempo real também poderia ser utilizada, de acordo com o preconizado por GRISSO et al. (2011), sugerindo que a dosagem de sementes seria definida a partir de dados de sensores de textura, eletrocondutividade ou matéria orgânica, mas não foram identificadas semeadoras do mercado atual com essa configuração.

Sistemas de controle para semeadura de precisão

Sistemas de controle são conjuntos de componentes eletrônicos, mecânicos e hidráulicos responsáveis pelo acionamento dos dosadores ou órgãos ativos de máquinas à TV, para que executem as prescrições correspondentes. $\mathrm{O}$ controle ocorre com ou sem mudança de dosagem, caracterizando os chamados regimes transitório e estacionário. As transições de dose decorrem de degraus de comando correspondentes à alteração da taxa de descarga das máquinas, que, de acordo com o tipo de sistema, irão ocorrer em mudanças de zonas de mapas, alterações das leituras dos sensores, de velocidade de trabalho ou comandadas pelo operador (GRISSO et al., 2011; OGATA, 2011).

Os sistemas de controle enviam sinais para os atuadores, que alteram a taxa de descarga dos equipamentos. No caso das semeadoras-adubadoras à TV, os componentes eletrônicos estão centralizados em um módulo do controlador montado junto ao terminal para interface com o usuário. $\mathrm{O}$ módulo central, na maioria dos sistemas comerciais, também dispõe da navegação por Sistema de Posicionamento Global Diferencial (DGPS), mas podem ser usados terminais independentes e outros sistemas de localização, de acordo com a exatidão espacial determinada pela operação. Pela interface, o usuário configura dados da 
máquina e da operação, insere mapas de semeadura e fertilização, e coleta dados de trabalhos realizados (BRAGACHINI, 2000; SHEARER et al., 2002).

Os sistemas de controle das semeadoras à TV monitoram a velocidade de trabalho do conjunto mecanizado por radar, sensores nos rodados das máquinas, ou mesmo aferição pelo sistema de localização. Os atuadores utilizados para acionar a rotação dos dosadores de sementes e fertilizantes, em maioria, são motores hidráulicos, mas podem ser utilizados motores elétricos. Sensores também monitoram a rotação dos eixos dos dosadores, na malha fechada, cujas correções autônomas desses sistemas promovem oscilações, que são consideradas normais para uma amplitude de 2 a $5 \%$ em torno das rotações médias, caracterizando a acomodação (BRAGACHINI, 2000; SHEARER et al., 2002; OGATA, 2011).

Nos sistemas hidráulicos, a pressão é gerada por uma ou duas bombas, com acoplamento na tomada de potência, sendo que a pressão pode também acionar turbinas para dosadores pneumáticos. O sistema possui um reservatório e um trocador de calor próprios. A pressão também pode ser fornecida diretamente pelo sistema hidráulico do trator, o que pode reduzir custos, mas necessita maior cuidado para atender plenamente a vazão do sistema da semeadora (BRAGACHINI, 2000; SHEARER et al., 2002).

Quanto aos dosadores das semeadoras em linha de precisão, podem ser de diferentes tipos, quaisquer deles aplicáveis em sistemas de controle à TV. As semeadoras em linha utilizam principalmente mecanismos do tipo discos alveolados ou do tipo pneumático. Eles têm a função de dosar as sementes a uma taxa pré-determinada e, segundo MURRAY et al. (2006), devem ser capazes de proporcionar espaçamentos uniformes, pois as culturas apresentam uma resposta de rendimento em função da uniformidade de distribuição de sementes ao longo da linha. Conforme MACHADO et al. (2005), o parâmetro que define a precisão do mecanismo dosador de sementes é a regularidade da distribuição longitudinal.

\section{Acurácia das semeadoras-adubadoras à TV}

Segundo SHEARER et al. (2002), a acurácia é a diferença percentual entre a aplicação meta, denominada dosagem pretendida ou prescrita, e a dose real obtida em cada ponto no campo, nas avaliações técnicas, sendo comumente denominada dosagem experimental. Os autores afirmaram que a acurácia é resultado da combinação do desempenho dos componentes dos sistemas de controle, do algoritmo, bem como da qualidade do processo de calibração realizado pelo usuário. Além disso, erros nas dosagens estão sendo significativos, tanto para aplicação de sólidos quanto de líquidos, têm impacto na renda dos produtores e não são plenamente conhecidos pelos fabricantes, assistência técnica e revendedores. Um referencial de valor máximo para a acurácia de 3\% foi determinado por CERRI (2001), critério pelo qual validou um equipamento para aplicação de calcário à TV, por um método de coletas do insumo em zonas homogêneas dos mapas, durante a aplicação.

SHEARER et al. (2002) organizaram recomendações para embasar uma nova norma de ensaio de controladores de distribuidores, destacandose pela introdução do conceito de ensaio com trocas de taxa, uma vez que as normas antecessoras somente consideravam doses constantes. Sua metodologia diferencia-se pelo uso de uma disposição de coletores em forma de matriz, captando os efeitos das transições no sentido longitudinal e transversal da distribuição. Para as taxas, recomendam as combinações entre zero, 50, 75 e $90 \%$ das doses máximas dos insumos.

Para a interpretação do desempenho de uma máquina à TV com base na acurácia, SCHUMANN et al. (2006) utilizaram a correlação entre as dosagens prescritas e experimentais, para avaliar duas configurações de sistemas de controle de um equipamento para aplicação de fertilizantes. As avaliações aprovaram apenas uma das opções para a distribuição de insumos por árvore individualizada (single-tree), em citricultura. Relacionado a um grupo de projetos para redução da lixiviação de nitratos pela atividade na Flórida, o objetivo era confirmar a capacidade de os equipamentos variarem ou suprimirem a dose a cada espaçamento entre plantas, operando a uma velocidade de $1,34 \mathrm{~m} \mathrm{~s}^{-1}$.

$\mathrm{Na}$ figura 1, foram demonstrados os desempenhos dos Sistemas 1 e 2, de acordo com SCHUMANN et al. (2006). Quanto ao Sistema 1, destaca-se o afastamento da linear em relação à reta de referência 1:1, inclinação inadequada de parâmetro 0,78 , e pouca variação explicada pela linear $(85 \%)$. A posição da linear indicou sobredosagens nas baixas taxas e subdosagens para as maiores doses aplicadas. Esse efeito foi gerado pela constante da equação, que determinou uma interseção da linear e da 1:1 no centro do gráfico, reduzindo a acurácia média, que, na dose de $420 \mathrm{~kg} \mathrm{ha}^{-1}$, foi de 5,06\%. Por esse motivo, a acurácia deve ser complementada pela sua amplitude, neste caso corroborando as afirmativas de inadequação do equipamento, por valores desde 1,53 a $30,32 \%$. O equipamento demonstrou 

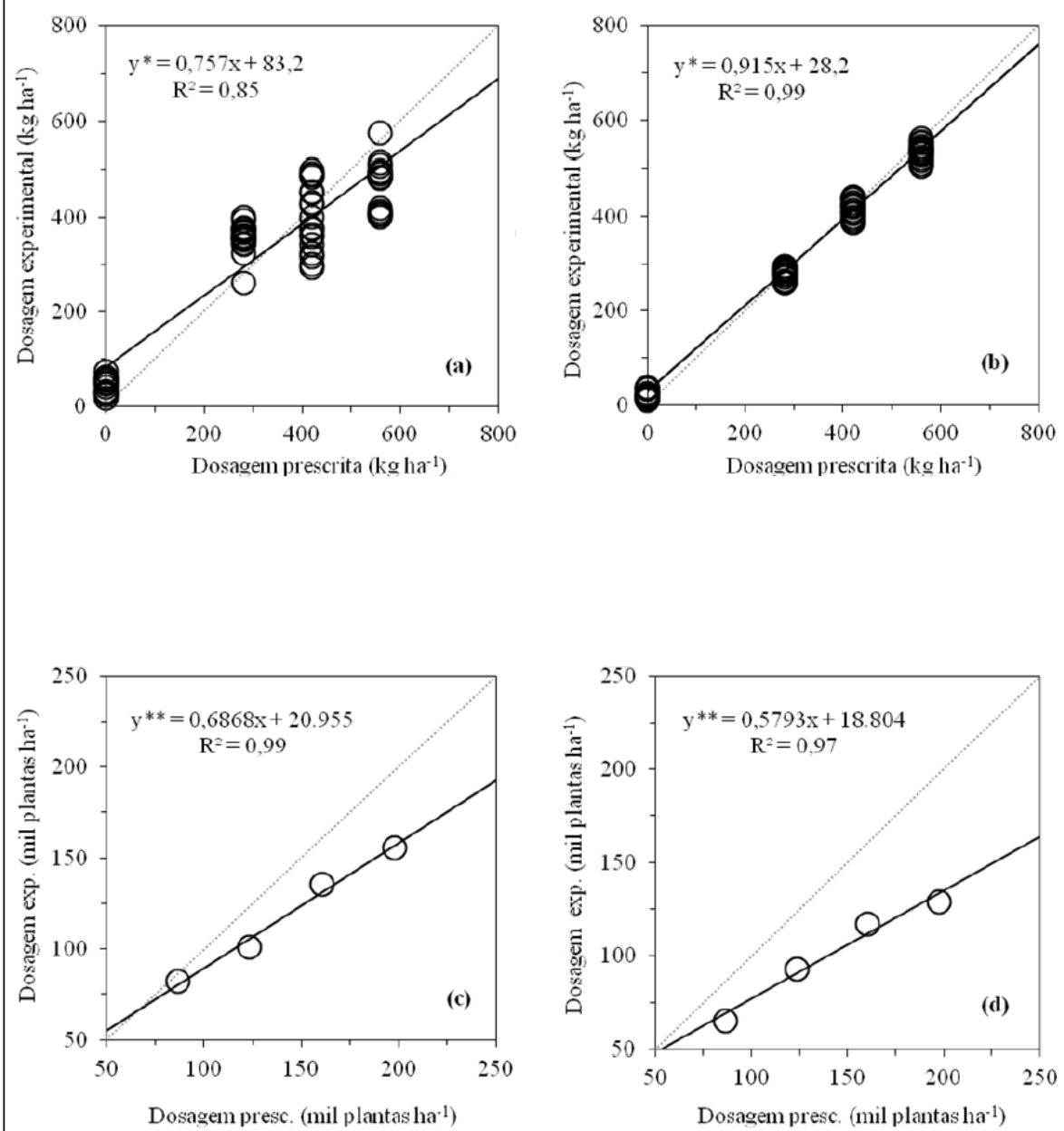

Figura 1 - Equações da acurácia de distribuidor centrífugo equipado com dois sistemas de controle (a e b) e de semeadora-adubadora à TV operando em duas avaliações na cultura do algodão (c e d).

Nota: (a) Sistema 1 e (b) Sistema 2 (SCHUMANN et al., 2006). (c) Cultivo de sequeiro e (d) Cultivo irrigado (FULTON et al., 2007). *Equações e coeficientes de determinação disponibilizados pelos autores. **Equações e coeficientes de determinação estimados pelos dados médios.

instabilidade, pelas distorções das trocas crescentes, das doses médias para a alta (50 e 75 para 90\%), que determinaram ocorrência de overshooting, que, segundo OGATA (2011), são picos de comando normalmente verificados no fenômeno transitório dos sistemas de controle. Os erros absolutos das dosagens experimentais atingiram de -160 a $120 \mathrm{~kg}$ $\mathrm{ha}^{-1}$, fazendo com que a acurácia atingisse até mais de $40 \%$. Para o Sistema 1, a distorção somente não foi maior no sentido da subdosagem, porque a constante da linear $83,2 \mathrm{~kg} \mathrm{ha}{ }^{-1}$ deslocou a intersecção das retas no sentido superior. Esse aspecto foi devido às aferições das dosagens ocorrerem de forma contínua, em uma simulação de 14 árvores, incluídas doses nulas. Ao reduzir a dosagem para zero, um retardo do sistema de controle provocou valores de dosagem nessa região do gráfico, onde a dose deveria ser nula, induzindo ao aumento do valor da constante da linear. O sistema 2 possui uma válvula alternativa para controle da dosagem e outro controlador no distribuidor. A operação teve desempenho consistente, sendo aprovado para a operação single-tree, com trocas de dosagem em menos de um segundo e acurácia adequada. A sobreposição das aferições na linear e o coeficiente angular de 0,915 fizeram com que as dosagens ocorressem próximas da reta 1:1. O nível de variabilidade foi aceitável, com $99 \%$ de variação explicada pela linear. A constante de apenas 
$28,2 \mathrm{~kg} \mathrm{ha}^{-1}$, demonstrou que a máquina rapidamente era capaz de suprimir a dose nos degraus decrescentes, ocorrendo pequenas interferências nas zonas nulas.

$\mathrm{Na}$ figura 1, destaca-se o desempenho de uma semeadora-adubadora à TV utilizada em dois experimentos, por FULTON et al. (2007). O desempenho da máquina é irregular e comparável ao Sistema 1, com inclinação inadequada das lineares e afastamento da reta 1:1. Os autores buscaram a validação de um possível acréscimo de produtividade ao adotar-se a semeadura à TV de algodão de sequeiro e irrigado, utilizando faixas de prescrições uniformes, com densidades de semeadura 86, 124, 161 e 198 mil plantas ha-1. Embora os autores não tenham visado à aprovação do equipamento, a acurácia superou o valor máximo recomendado, com média $15,06 \%$ para a Avaliação 1 e $27,75 \%$ para a Avaliação 2, sendo o maior valor para a segunda, devido ao menor parâmetro de inclinação. Os autores afirmaram que os erros negativos podem estar ligados a possíveis problemas na calibração e configuração da máquina, associados com baixas germinação e emergência. Entretanto, estima-se que os desvios sejam sistemáticos e proporcionais com o aumento da dosagem, mantendo a proximidade com as lineares, o que é atribuído ao controlador, pois efeitos do ambiente ou sementes reduziriam o coeficiente de determinação, o que não foi observado.

Embora o experimento de cultivo de algodão de sequeiro tenha apresentado maior aproximação das metas, em que o melhor desempenho ocorreu na menor dosagem, com acurácia média de $5 \%$, essa informação não indica adequação operacional, devido à ausência de dados de dispersão das populações de plantas de algodão aferidas no campo. Nas demais dosagens, a acurácia passou de 5,00 para 18,$25 ; 15,67$ e $21,32 \%$. No cultivo irrigado, a acurácia passou de 24,41, para 24,64; 27, 18 e 34,75\%. Quanto à estabilidade operacional da semeadora avaliada por FULTON et al. (2007), os coeficientes de determinação de 99 e $97 \%$ são compatíveis ao Sistema 2 de SCHUMANN et al. (2006), com valor 99\%. Entretanto, com apenas dados médios, não é possível comprovar se proximidade dos marcadores ao longo da linear foi efetiva.

Corroborando a ocorrência de erros negativos na semeadura à $\mathrm{TV}$, dados de 47 testes de lavouras de milho implantadas com máquinas de produtores rurais norte-americanos, convencionais e à TV, apresentados por REEG (2011), identificaram populações no campo inferiores às metas. Ao estratificar as aferições em dois níveis de erros, verificaram que, no primeiro, com desvio de até
2.471 sementes ha $^{-1}$ (aproximadamente acurácia $3 \%$ ), estavam compreendidas $54,50 \%$ das lavouras avaliadas. O outro nível de desvio, de 4.942 sementes $\mathrm{ha}^{-1}$, somou $21,00 \%$ das avaliações.

Os erros negativos também podem ser verificados em experimentos desenvolvidos por grupos de pesquisa, demonstrando que não ocorreram somente em avaliações de cooperativas e em lavouras comerciais, como as anteriores. Os exemplos de HÖRBE et al. (2013), na cultura do milho, e UHRY (2013), na cultura da soja, também não foram focados no desempenho da máquina, embora os dados disponibilizados pelo primeiro tenham incluído os intervalos de confiança para as médias das populações aferidas no campo.

No estudo de HÖRBE et al. (2013), os objetivos visaram a definir as possibilidades de otimização da produtividade, em função da densidade da semeadura para três zonas de manejo. Foram realizadas duas avaliações, denominadas Experimento 1 e Experimento 2, que corresponderam a dois ciclos agrícolas subsequentes, sendo verificados contrastes entre as populações obtidas para as mesmas prescrições. Embora ambos com erros absolutos negativos, no Experimento 1, a acurácia manteve-se no limite referencial, com valores desde 0,37 a 2,92, e média $1,50 \%$. No Experimento 2 a acurácia média foi de 9,26 , variando entre 7,08 a $12,38 \%$.

As populações médias aferidas por HÖRBE et al. (2013) podem ser verificadas na figura 2. A linear do Experimento 1 está próxima da 1:1, corroborando a verificação de acurácia adequada pela inclinação de 0,95 , demonstrando uma pequena interferência, no sentido de subdosagens, com o aumento da população. O parâmetro de inclinação é semelhante ao Sistema 2, aprovado por SCHUMANN et al. (2006). No Experimento 2, o parâmetro é inferior à referência, mas superior à semeadora utilizada por FULTON et al. (2007), o que indica que o desempenho foi mais comprometido pelo deslocamento da linear abaixo da reta $1: 1$, do que propriamente pela inclinação da reta. As deficiências da população foram atribuídas pelos autores aos efeitos de chuvas excessivas após a semeadura, elevando a acurácia para 7,08\%, em relação ao valor 0,37\% do Experimento 1 .

Quanto à variabilidade da dosagem, os coeficientes de determinação verificados por HÖRBE et al. (2013), ambos 99\%, equiparam-se ao Sistema 2 de SCHUMANN et al. (2006), bem como com as avaliações de FULTON et al. (2007), com 99 e 97\%. Entretanto, os gráficos disponibilizados por HÖRBE et al. (2013) permitiram uma estimativa dos intervalos de confiança. 

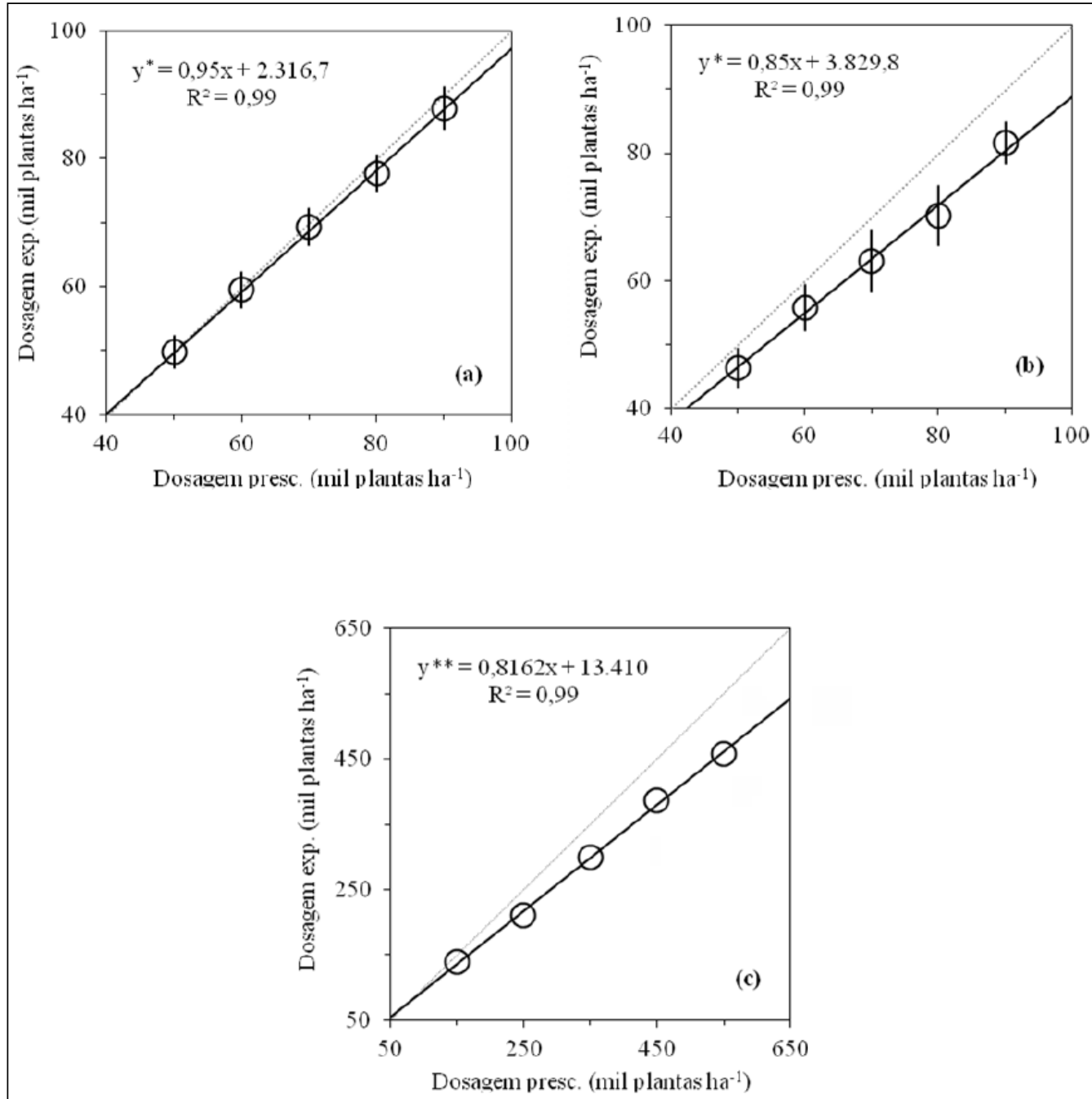

Figura 2 - Equações da acurácia de semeadoras-adubadoras à TV na implantação da cultura do milho (a e b) e da soja (c).

Nota: (a) Experimento 1 e (b) Experimento 2 (HÖRBE et al., 2013). (c) UHRY (2013). *Equações e coeficientes de determinação disponibilizados pelos autores. **Equações e coeficientes de determinação estimados pelos dados médios.

Embora HÖRBE et al. (2013) tenham afirmado que as intempéries causaram as interferências entre os experimentos, a disposição das médias próximas das lineares pode indicar a ocorrência concomitante de efeitos sistemáticos. Ao analisar a qualidade da distribuição de sementes, que foi avaliada para o Experimento 2, verificase a manutenção de altos índices de espaçamentos aceitáveis, na ordem de 89 a 94\%, reforçando efeitos sistemáticos e não aleatórios, que seriam aqueles envolvendo germinação e efeitos do ambiente. O coeficiente de variação (CV) médio foi de 14,20\%. Esse desempenho foi superior aos limites mínimos recomendados, tanto para dosadores de discos alveolados quanto pneumáticos, respectivamente, de 50 e $90 \%$ de aceitáveis, e 50 e $30 \%$ para o CV, estipulados por COELHO (1996). HÖRBE et al. (2013) não especificaram o tipo de dosador que equipou a semeadora durante as avaliações.

Para a cultura da soja, o experimento realizado por UHRY (2013) também comprovou a ocorrência de erros absolutos negativos. Os dados 
representados no gráfico de correlação, disposto na figura 2, cujas dosagens prescritas foram de 150 , 250, 350, 450 e 550 mil plantas ha ${ }^{-1}$, demonstraram aumento progressivo dos erros das aferições no sentido negativo, com média -50,9 e amplitude desde -11 a -91 mil plantas ha-1 ${ }^{-1}$ A acurácia média foi de 13,59; aferindo-se desde 7,33 a 16,55\%. Para a menor dosagem, a acurácia equiparou-se ao Experimento 2 de HÖRBE et al. (2013), mas o menor parâmetro angular de 0,8162 promoveu subdosagens mais severas. À semelhança das discussões anteriores, não foi visada validação da máquina, embora tenha extrapolado a acurácia máxima tolerável. Os pontos médios, em semelhança às Avaliações 1 e 2 e do Experimento 2, apesar de pouca variabilidade em relação à linear, com coeficiente de determinação de $99 \%$, não permitiram identificar possíveis oscilações e inadequações das dosagens.

\section{CONCLUSÃO}

As semeadoras estão operando, em maioria, com acurácia acima do limite preconizado, pois, dentre os experimentos identificados, apenas em um destes a acurácia não excedeu 3\%, o que reprovaria $80 \%$ dessas operações à TV.

As causas do desempenho insuficiente atribuem-se a efeitos sistemáticos do algoritmo e da calibração dos equipamentos, que determinaram deflexões excessivas nas lineares das dosagens experimentais, além de possíveis efeitos de germinação e emergência. $O$ desempenho das semeadoras caracterizou-se pela implantação das culturas com subdosagens crescentes ao longo das prescrições, atingindo acurácia de até 34,75\%.

A análise sistemática da acurácia foi eficaz em identificar os fatores responsáveis pela distorção do desempenho das máquinas, permitindo qualificar o desempenho das semeadoras-adubadoras à TV e obter comparações entre os equipamentos.

\section{REFERÊNCIAS}

ANDRADE-SANCHEZ, P.; HEUN, J. Understanding technical terms and acronyms used in Precision Agriculture. Arizona Cooperative Extension, Tucson, 2010. Disponível em: <http:// arizona.openrepository.com/arizona/handle/10150/146427>. Acesso em: 05 mar. 2012.

AUERNHAMMER, H. Precision farming - the environmental challenge. Computers and Electronics in Agriculture, v.30, p.31-43, 2001.

BERNARDI, A.C. de C. et al. Aplicação de fertilizantes a taxas variáveis. In: MACHADO, P.L.O. de A. et al. Agricultura de precisão para o manejo da fertilidade do solo em sistema plantio direto. Rio de Janeiro: Embrapa Solos, 2004. p.153-164.
BRAGACHINI, M. et al. Tecnologia de aplicación variable de insumos. Córdoba: INTA Manfredi, 2000. (PROYECTO AGRICULTURA DE PRECISIÓN).

CERRI, D.G.P. Desenvolvimento de um sistema de aplicação localizada de calcário a taxas variáveis. 2001. 90f. Dissertação (Mestrado em Agronomia) - Universidade de São Paulo, São Paulo, SP.

COELHO, J.L.D. Ensaio \& certificação das máquinas para a semeadura. In: MIALHE, L.G. Máquinas agrícolas: ensaio \& certificação. Piracicaba: Fundação de Estudos Agrários Luiz de Queiroz, 1996. p.551-569.

FULTON, J.P. et al. Comparison of variable-rate granular application equipment. In: ASAE ANNUAL INTERNATIONAL MEETING, 2003, Las Vegas. Proceedings... Las Vegas: ASAE, 2003. p.1-15.

FULTON, J.P. et al. Evaluation of variable-rate seeding for cotton. Auburn: Alabama Agricultural Experimental Station, 2007. (2006 COTTON RESEARCH REPORT).

FULTON, J.P. Overview of variable-rate technology. Alabama: Alabama Precision Ag Extension, 2009a. (Precision Agriculture Series).

FULTON, J.P. Variable-rate application (VRA): does it works? Alabama: Alabama Cooperative Extension System. 2009b. (PRECISION AGRICULTURE INCENTIVE TRAINING).

FULTON, J.P. et al. Proper implementation of precision agricultural technologies for conducting on-farm research. In: INTERNATIONAL CONFERENCE ON PRECISION AGRICUlTURE, 10., 2010, Denver. Proceedings... Denver: International Society of Precision Agriculture, 2010. p.1-3.

GEBHARDT, M.R. et al. Automatic sprayer control system. Transactions of ASAE, v.17, n.6, p.1043-1047, 1974.

GRISSO, R. et al. Precision farming: a comprehensive approach. Blacksburg: Virginia Tech, 2009. (Virginia Cooperative Extension).

GRISSO, R. et al. Precision farming tools: variable-rate application. Blacksburg: Virginia Tech, 2011. (Virginia Cooperative Extension).

HAAG, L.A.; STAGGENBORG, S.A. Variable rate corn seeding based on spatially variable growing season water supply. In: AMERICAN SOCIETY OF AGRONOMY INTERNATIONAL MEETINGS, 2006, Indianápolis. Proceedings... Indianápolis: American Society of Agronomy, 2006. p.1-15.

HÖRBE, T. de A.N et al. Optimization of corn plant population according to management zones in Southern Brazil. Precision Agriculture, v.14, p.450-465, 2013. Disponível em: <http://www. researchgate.net/publication/258833904_Optimization_of_corn_ plant_population_according_to_management_zones_in_Southern_Brazil>. Acesso em: 21 jan. 2014. doi: 10.1007/s11119-013-9308-7.

MACHADO, A.L.T. et al. Máquinas para preparo do solo, semeadura, adubação e tratamentos culturais. 2.ed. Pelotas: UFPEL, 2005. 253p.

MANTOVANI, E.C. Agricultura de precisão e sua organização no Brasil. In: BORÉM, A. et al. (Orgs.). Agricultura de precisão. Viçosa: UFV, 2000. p.77-92. 
MÉNDEZ, A. Agricultura de precisión y máquinas precisas. Córdoba: INTA Manfredi, 2011. (PROYECTO AGRICULTURA DE PRECISIÓN).

MIALHE, L.G. Máquinas agrícolas para plantio. Campinas: Millennium, 2012. 623p.

MOLIN, J.P. et al. Avaliação de intervenções em unidades de aplicação localizada de fertilizantes e de populações de milho. Engenharia Agrícola, v.26, n.2, p.528-536, 2006. Disponível em: $<$ http://www.scielo.br/scielo.php?script=sci arttext\&pid=S0100$69162006000200022 \& \operatorname{lng}=\mathrm{pt} \& \mathrm{nrm}=\mathrm{iso}>$. Acesso em: 21 jan. 2014. doi: $10.1590 / \mathrm{S} 0100-69162006000200022$.

MURRAY, J.R. et al. Planters and their components: types, attributes, functional requirements, classification and description. Canberra: Australian Centre for International Agricultural Research. 2006, 178p.

OGATA, K. Engenharia de controle moderno. 5.ed. São Paulo: Saraiva, 2011. 824p.

PASZKIEWICZ, S.; BUTZEN, S. Corn hybrid response to plant population. Pioneer Crop Insights, v.17, n.16, p.1-4, 2007.

PING, J.L. et al. Site-specific nitrogen and plant density management in irrigated maize. Agronomy Journal, v.100, n.4, p.1193-1294, 2008 Disponível em: $<$ http://hybridmaize.unl.edu/assets/publications/pdf453. PDF>. Acesso em 28 dez. 2012. doi: 10.2134/agronj2007.0174.

REEG, P. Corn plant population and variable rate planting. In: ONFARM NETWORK CONFERENCE, 2011, Ames. Proceedings... Ankeny: Iowa Soybean Association, 2011, p.1-65.
SCHUELLER, J.K. Impediments to spatially-variable field operations. Computers and Electronics in Agriculture, v.14, p.249-253, 1996. Disponível em: <http://www.sciencedirect.com/ science/article/pii/0168169995000518>. Acesso em 17 jun. 2013. doi: 10.1016/0168-1699(95)00051-8.

SCHUMANN, A.W. et al. Optimizing variable rate granular fertilizer spreader performance for single-tree prescription zones. In: ASAE ANNUAL INTERNATIONAL MEETING, 2006, Portland. Proceedings... Portland: ASABE, 2006. p.1-14.

SHEARER, S.A. et al. Considerations for development of variable-rate controller test standard. In: ASAE ANNUAL INTERnATIONAL MEeting, 2002, Chicago. Proceedings... Chicago: ASAE e CGIR, 2002. p.1-14.

STAFFORD, J.V. Implementing precision agriculture in the 21 st century. Journal of Agricultural Engineering Research, v.76, p.267-275, 2000. Disponível em: $<$ http://www.rmorais.utad.pt/RMPDFFiles/PDFPapers/ STAFFORD00.pdf $>$. Acesso em 26 jun. 2013. doi: 10.1006/ jaer.2000.0577.

UHRY, D. Avaliação e critério para utilização de semeadora com sistema de taxa variável de sementes na cultura da soja. 2013. 141f. Tese (Doutorado em Engenharia Agrícola) - Universidade Federal de Santa Maria, Santa Maria, RS.

VIDRINE, C.G. et al. A constant pesticide application rate sprayer model. Transactions of ASAE, v.18, n.3, p.439-443, 1975. 